Recent Insights into the Physics of the Sun and Heliosphere:

Highlights from SOHO and Other Space Missions

IAU Symposium, Vol. 203, 2001

P. Brekke, B. Fleck, and J. B. Gurman eds.

\title{
Active Region Evolution and Flare Activities: From the Photosphere to the Corona
}

\author{
T. T. Ishii and H. Kurokawa \\ Kwasan and Hida Observatories, Kyoto University, Yamashina-ku, \\ Kyoto, JAPAN, 607-8471 \\ T. T. Takeuchi \\ Division of Particle and Astrophysical Sciences, Nagoya University, \\ Nagoya, JAPAN, 464-8602
}

\begin{abstract}
.
We studied the evolution of an active region and its relation with flare activities in order to solve the physical mechanism of solar flare occurrence. We investigated the evolutionary characteristics of the active region NOAA 8948 (April 2000) and found that the newly flux emergence caused the flares in this active region.
\end{abstract}

\section{Introduction}

The formation process of magnetic shear is a key to understand the physical mechanism of solar flare occurrence. In the previous works, we suggest that the emergence of a twisted flux bundle should be the key to the high flareproductivity of the sunspot group, or the flare energy build-up mechanism (Ishii et al. 1998, 2000). In order to examine such a process, we need observational data of active region evolution from the photosphere to the corona.

\section{Observational Data}

In this paper, we study the active region evolution and its relation with flare activities in the active region NOAA 8948 (April 2000). We use $\mathrm{H} \alpha$ images obtained at Hida and Kwasan Observatories and white light images by Transition Region And Coronal Explorer (TRACE). We also use the Solar and Heliospheric Observatory (SOHO)/ Michelson Doppler Imager (MDI) intensitygram and longitudinal magnetogram for the investigation of photospheric structures of the active regions. Coronal structures of the regions are studied by using extreme ultraviolet (EUV) images obtained with SOHO and TRACE. 


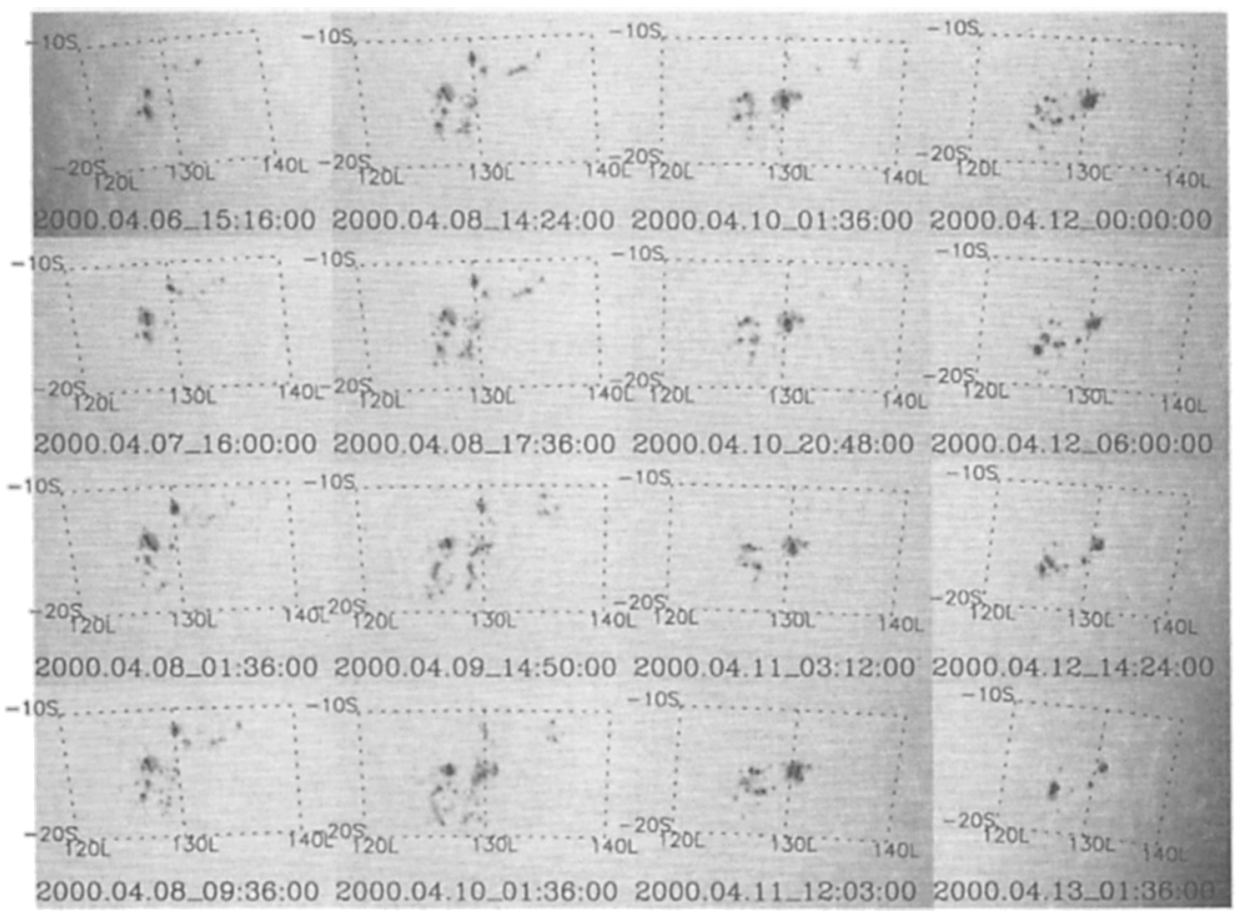

Figure 1. Daily evolution of the active region NOAA 8948. SOHO/MDI intensitygrams. $S$ : latidue, $L$ : Carrington longitude.

\section{Results}

\subsection{Active Region Evolution}

The daily evolution of the active region NOAA 8948 (Fig. 1) is summarized as follows:

1. Apr. 6: A bipolar pair of sunspots already existed.

2. Apr. 8 - 10: A bipolar pair of sunspots newly appeared, evolved and decayed (phase 1).

3. Apr. $11-13$ : Another bipolar pair of sunspots newly appeared, evolved and decayes (phase 2).

\subsection{Flare Activities}

Many $\mathrm{H} \alpha$ sub-flares and several X-ray (C- and M-class) flares occurred in this active region. Most of these flares occurred in the evolutionary phase 1 and 2. In figure 2, we found that the location of these flares are the same as the emerging flux regions in both evolutionray phases. Comparing the TRACE $171 \AA$ images in the right panels of Fig. 2, we can clearly see that the M1.3 flare occurred just in the emerging flux region in phase 2. 

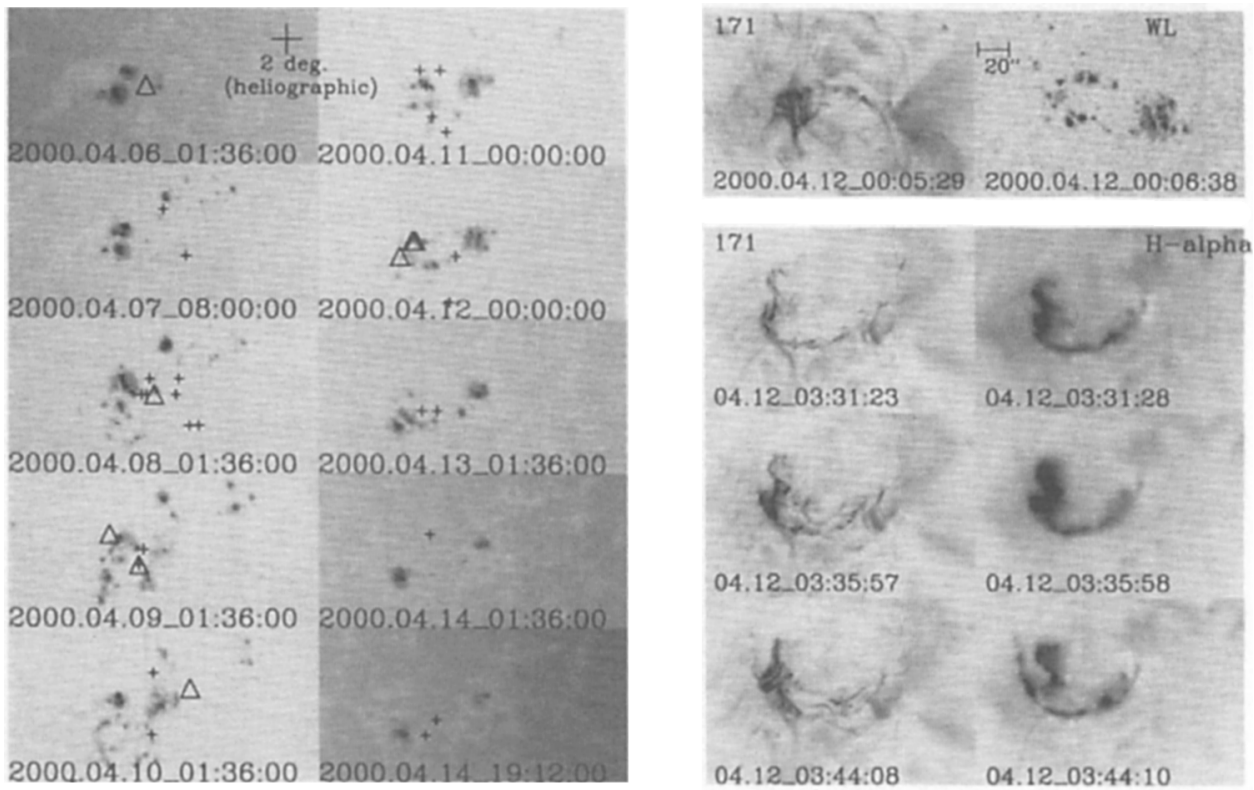

Figure 2. Flares in the active region NOAA 8948. Left: Daily, Mclass (triangle) and C-class (plus sign) flares. Right: M1.3 flare in the emerging flux region on April 12.

\section{Summary}

We summarize the relation between the active region evolution and flare activities as follows:

1. Magnetic flux emergence formed a bipolar pair of sunspots.

2. Flares occurred in the emerging flux region.

3. After showing flare-acitivity, the sunspots decayed.

Acknowledgments. We made extensive use of National Solar Observatory Digital Library and the Space Physics Interactive Data Resource (SPIDR).

\section{References}

Ishii, T. T., Kurokawa, H., \& Takeuchi T. T. 1998, ApJ, 499, 898

Ishii, T. T., Kurokawa, H., \& Takeuchi T. T. 2000, PASJ, 52, 337 